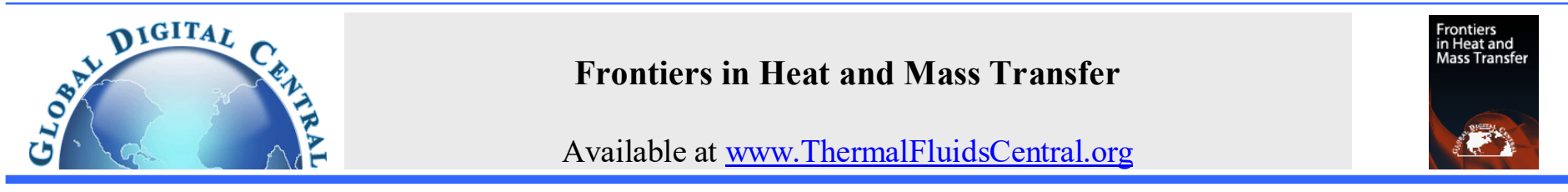

\title{
DRUG RELEASE IN TRANSDERMAL DEVICES: A BRIEF PARAMETRIC STUDY
}

\author{
F.C.C. Almeida ${ }^{\mathrm{a}}$, P.M. da Silva ${ }^{\mathrm{b}, \mathrm{c}}$, M.N.C. Coelho Pinheiro, ${ }^{\mathrm{a}, \mathrm{d},{ }^{*}}$ \\ a Instituto Superior de Engenharia do Instituto Politécnico de Coimbra, Departamento de Engenharia Química e Biológica, Rua Pedro Nunes, \\ Quinta da Nora, 3030-199 Coimbra, Portugal \\ ${ }^{b}$ Instituto Superior de Engenharia do Instituto Politécnico de Coimbra, Departamento de Física e Matemática, Rua Pedro Nunes, Quinta da Nora, \\ 3030-199 Coimbra, Portugal \\ ${ }^{c}$ Centro de Matemática da Universidade de Coimbra, Apartado 3008, EC Santa Cruz,3001 - 501 Coimbra, Portugal \\ ${ }^{d}$ Centro de Estudos de Fenómenos de Transporte, Faculdade de Engenharia da Universidade do Porto, Rua Dr. Roberto Frias, 4200-465 Porto,
} Portugal

\begin{abstract}
In the last decades, polymeric systems were essayed and applied successfully as vehicles for transdermal delivery devices of active principles. Applications in contraception, pain control, and smoking cessation are already frequent. A crucial aspect in the development of new formulations is the maintenance of the therapeutic drug concentration range for long periods. Recognizing this topic as interesting and challenging, simple models to describe drug release from a transdermal device were developed with the purpose to perform a parametric sensitivity analysis. Assuming drug diffusion within the polymeric delivery system as the dominant mechanism the models were developed and implemented. Transdermal patches made of the single adhesive material layer (the drug reservoir) and made of two layers were considered during drug release modeling to the skin. The sensitivity analysis of parameters such as drug diffusion coefficient and initial drug concentration brought insight into drug transfer process inside the patches, revealing itself as a useful tool in the optimization of drug delivery devices.
\end{abstract}

Keywords: Drug delivery; Transdermal drug device; Drug release profiles; Parametric exploration

\section{INTRODUCTION}

Transdermal delivery systems releasing active principles for pain control, hormone replacement and conception prevention have been increasingly accepted over the last decades. Another successful application is nicotine replacement in smoking cessation treatment of dependent smokers. In fact, this way of drug administration is non-invasive, can be selfadministered and generally is inexpensive and consequently improves the patient compliance in the treatments.

Polymeric matrices have been used largely as more efficient vehicles for drug delivery to the intended local and in particular in transdermal devices. Pharmacokinetics must be appropriate to maintain the therapeutic drug concentration range during long periods and drug dissolution profile assessment are used as quality parameter in routine quality control during delivery system production, as well as, in the development of new formulations or optimization of existing ones.

Scientific community while studying and modelling the medication transfer process throughout the delivery devices and the consequent release to the neighboring environment drove the technological improvement in drug delivery vehicles. Several studies have been publishing, proposing new and more accurate models describing transdermal drug release and the skin barrier (Anissimov et al. (2013), Frasch and Barbero (2013), Jepps et al. (2013), Kalia and Guy (2001), Khanday and Rafiq (2015), Snorradõttir et al. (2014)).

This paper reports a real problem of drug release describing mathematically the physical situation in transdermal devices. After the code development to obtain the mathematical solution a parametric sensitivity analyses was performed.

Transdermal patches made of a single layer, that acts as drug reservoir, and made of two different layers were considered for modelling the drug release to the skin. The code, in MATLAB, developed to perform mass transfer calculations, allowed exploring the effect on drug release kinetics of model key parameters as: diffusion coefficient and initial drug concentration distribution in multiple layer patches. Parametric studies are essential in the design/optimization of drug delivery devices diminishing the necessity of in vitro and in vivo studies in large scale.

\section{THE PHYSICAL SITUATION}

Drug transport through polymeric devices is usually described as a nonsteady state diffusion problem when structural modifications in solid matrices are negligible. With drug diffusion as the controlling mechanism for mass transfer, the Fick's second law of diffusion can be used

$\frac{\partial C_{A}}{\partial t}=-\nabla \bullet\left(-D \nabla C_{A}\right)$

where $C_{A}$ represents the drug concentration in the solid matrix, $D$ stands for the drug diffusion coefficient and $t$ represents the time. Assuming constant drug diffusion coefficient, the last equation is given by

\footnotetext{
*Corresponding author.Email: mnazare@isec.pt
} 
$\frac{\partial C_{A}}{\partial t}=D\left(\nabla \bullet \nabla C_{A}\right)=D \nabla^{2} C_{A}$

and for Cartesian coordinates $(x, y, z)$ the following equation is considered,

$$
\frac{\partial C_{A}}{\partial t}=D\left(\frac{\partial^{2} C_{A}}{\partial x^{2}}+\frac{\partial^{2} C_{A}}{\partial y^{2}}+\frac{\partial^{2} C_{A}}{\partial z^{2}}\right)
$$

Models have been developed in order to complement experimentation, in which concerns drug delivery of drugs, or others bioactive substances, from polymer based devices. Transdermal drug delivery is an important application where polymeric planar patches with an active principle incorporated are used to release therapeutic doses, during long periods, to adjacent tissues to the local of application in the body (topical release) or to the sanguine flow after crossing the skin barrier (systemic release). Understand the biotransport process throughout the polymeric delivery device is of crucial importance as this "acts as a drug reservoir, and an optimal design of its microstructural characteristics would improve the release performances" (Pontrelli and de Monte, 2014; Rim et al. 2005).

Frequently the amount of drug loaded in the patch is lower than the solubility of the active principle in the polymeric matrix and only dissolved drug exists. Without solid drug coexisting, there is no need to consider the rate of dissolution in the polymeric device and Fick's second law for diffusion is enough to describe drug transport process.

\section{THE MATHEMATICAL APPROACH}

The skin is the human body greatest organ with a high complexity and vital protection, regulation and sensorial functions. Structural and functional differences are observed with skin ageing, body site, between human races (Williams (2003)), among many others factors, causing modifications in drug absorption. Although, in transdermal drug delivery commonly the human skin is considered divided in three main layers: epidermis, dermis and hypodermis, from the outermost to the innermost layer. The epidermis is itself a multi-layered membrane encompassing the stratum corneum, stratum granulosum, stratum spinosum and the stratum germinativum (Williams (2003)). The external layer, the stratum corneum, consisting of keratinized cells embedded in a lipid matrix constitutes the principal barrier in the transdermal delivery of drugs.

The complexity and diversity of skin structure make modelling penetration and distribution of the drug absorbed by skin very difficult. The simplest mathematical approach to describe drug transport through the stratum corneum layer considers it as a homogeneous membrane with a medication donor phase (with uniform and constant concentration) at the top and a rapid drug clearance process at the lower boundary, maintaining there a location of zero drug concentration (Anissimov et al. (2013), Jepps et al. (2013)). Although in the last decades increasingly refined models appeared with intention to access the drug transdermal transport with more detail, they are not suitable for consideration when a diligent and effective application is required in preliminarily studies. For this reason, the barrier of skin is not considered (explicitly) in the present mathematical approach developed. However, care was taken to emphasize that in light of the simplest skin modelling transport process this is comparable to have an extra layer in the polymeric patch.

\subsection{Transdermal drug device: mono-layered patch}

Transdermal drug delivery systems, as (flat) patches made of adhesive polymeric materials with drug incorporated, can be approximated to onedimensional problem with the significant drug concentration gradient in the normal direction, $z$, to the skin, as illustrated in Fig. 1. In this situation, Eq. (3) is simplified to

$\frac{\partial C_{A}}{\partial t}=D \frac{\partial^{2} C_{A}}{\partial z^{2}}$

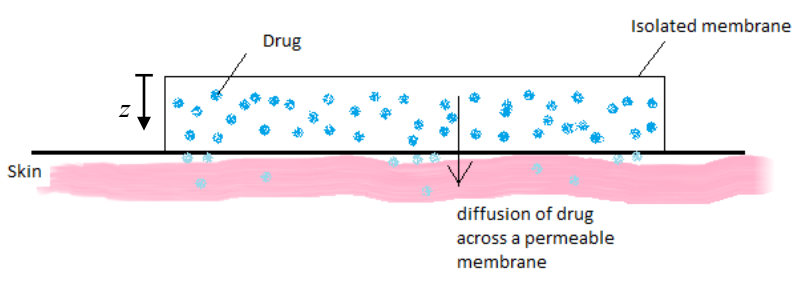

Fig. 1 Sketch of an adhesive patch with drug incorporated, as an example of a transdermal drug device.

Solving this differential equation with the appropriate boundary and initial conditions for the transdermal delivery system it is possible to obtain drug concentration profiles inside the polymeric matrix. The profile equation gives the spatial (along $z$ ) and temporal drug concentration distribution throughout the patch, which serves as a drug reservoir.

Considering a flat patch made of a single layer, coated with an adhesive, where the drug is uniformly distributed, the following initial condition can be used

$C_{A}(z, t=0)=C_{A i} \quad, \quad z \in[0, \ell]$

where $C_{A i}$ represents the initial drug concentration in the delivery device. When the patch, with thickness $\ell$, is applied over an absorbing surface as skin, the following boundary conditions can be considered

$-D \frac{\partial C_{A}}{\partial z}(z=0, t)=0 \quad, \quad t>0$

if the coating adhesive is impermeable to the drug and

$C_{A}(z=\ell, t)=C_{A s} \quad, \quad t>0$

if the drug concentration in the patch surface contacting the skin is assumed constant and equal to $C_{A s}$.

Equation (6) indicates that drug flux through the upper patch surface, at $z=0$, is null as the adhesive was considered impermeable and represents the well-known Newmann boundary condition. Equation (7) specifies the drug concentration in the patch polymeric layer at $z=\ell$, and it is known as a Dirichlet boundary condition. The concentration $C_{A s}$ is assumed to be virtually zero, as a first approximation, to resolve the unsteady state diffusion problem for drug throughout the polymeric patch. This assumes that drug permeation through skin is not the rate limiting process and medication delivered from the patch is rapidly absorbed into bloodstream, which is a rough assumption.

Solving (analytical or numerically) the problem described by the partial differential equation and the initial and boundary conditions it is possible to obtain the drug distribution within the transdermal device in each instant $t$ of the delivery process. Then, the amount of medication inside the patch with a single layer, $M(t)$, in each instant $t$, can be calculated using

$M(t)=A \int_{0}^{\ell} C_{A}(z, t) d z$

where $A$ is the polymeric patch area.

In pharmacokinetics is of great importance to know the amount of medication released from the patch during a period of time, which can be obtained by subtraction of Eq. (8) from the initial amount of medication in the patch, $M_{i}$. Thus, 


$$
M_{i}-M(t)=A\left[C_{A i} \ell-\int_{0}^{\ell} C_{A}(z, t) d z\right],
$$

can be defined for single layer patches.

\subsection{Transdermal drug device: double-layered patch}

If a double-layered patch is considered as a solution to obtain a safe and controlled drug delivery system, the mathematical approach presented before must be extended in order to include two different mediums for medication diffusion. Hence, two partial differential equations, one for each different medium, similar to Eq. (3) must be used,

$$
\left.\frac{\partial \bar{C}_{A}}{\partial t}=\bar{D} \frac{\partial^{2} \bar{C}_{A}}{\partial z^{2}} \quad, \quad z \in\right] 0, \ell_{1}[, t>0
$$

for layer 1 with thickness $\ell_{1}$ and

$$
\left.\frac{\partial \overline{\bar{C}}_{A}}{\partial t}=\overline{\bar{D}} \frac{\partial^{2} \overline{\bar{C}}_{A}}{\partial z^{2}} \quad, \quad z \in\right] \ell_{1}, \ell_{2}[, t>0
$$

for layer 2 with thickness $\left(\ell_{2}-\ell_{1}\right)$, which is in contact with skin. The total thickness of the patch is $\ell_{2}$. To represent variables (such as drug concentration and diffusion coefficient) for layer 1 and layer 2, a single bar and a double bar above was used respectively, as can be seen in Eqs. (10) and (11).

If initially, the diffusing specie is uniformly distributed through patch polymeric layers, the following equations

$$
\begin{aligned}
& \bar{C}_{A}(z, t=0)=\bar{C}_{A i}, \quad z \in\left[0, \ell_{1}\right] \\
& \left.\left.\overline{\bar{C}}_{A}(z, t=0)=\overline{\bar{C}}_{A i}, \quad z \in\right] \ell_{1}, \ell_{2}\right]
\end{aligned}
$$

can be used as initial conditions, for layer 1 and layer 2, respectively. It should be emphasized that $\bar{C}_{A i}$ and $\overline{\bar{C}}_{A i}$ stands for the medication concentration (uniformly) distributed throughout layer 1 and 2 that constitute the double-layered patch. The two initial drug concentrations, in general, have different values and, in a limit situation, initially layer 2 can be free of medication, representing an additional barrier to mass transfer.

In the present situation, beyond two boundary conditions it is also necessary write one condition for the interface between layer 1 and 2 . The conditions for upper $(z=0)$ and lower $\left(z=\ell_{2}\right)$ boundary are expressed by equations similar to the ones used for the single layer patch as

$$
-\bar{D} \frac{\partial \bar{C}_{A}}{\partial z}(z=0, t)=0 \quad, \quad t>0
$$

and

$$
\overline{\bar{C}}_{A}\left(z=\ell_{2}, t\right)=\overline{\bar{C}}_{A s}, \quad t>0
$$

respectively. At the interface between layer 1 and 2 it is assumed the equation

$$
\bar{D} \frac{\partial \bar{C}_{A}}{\partial z}\left(z=\ell_{1}, t\right)=\overline{\bar{D}} \frac{\partial \overline{\bar{C}}_{A}}{\partial z}\left(z=\ell_{1}, t\right) \quad, \quad t>0
$$

which states that the drug flux coming from layer 1 should be equal to the flux going to layer 2 , and the equation

$$
\bar{C}_{A}\left(z=\ell_{1}, t\right)=k \overline{\bar{C}}_{A}\left(z=\ell_{1}, t\right) \quad, \quad t>0
$$

which denotes the equilibrium established for the drug distributed between layers, at the interface, and $k$ is the drug partition coefficient.

With the solution for the problem described by the partial differential equations and the initial and boundary conditions it is possible to obtain the amount of medication inside the patch, $M(t)$, in each instant $t$, by integration of concentration profiles over the two layers using

$$
M(t)=A\left[\int_{0}^{\ell_{1}} \bar{C}_{A}(z, t) d z+\int_{\ell_{1}}^{\ell_{2}} \bar{C}_{A}(z, t) d z\right]
$$

where $A$ is the polymeric patch area and the first and second integrals give the amount of drug within layer 1 and layer 2, by unit area, respectively. Subtracting Eq. (18) from the initial amount of medication in the patch, $M_{i}$, the amount of drug released during a period of time $t$ can be obtained for a double-layered patch

$$
M_{i}-M(t)=A\left[\bar{C}_{A i} \ell_{1}+\overline{\bar{C}}_{A i}\left(\ell_{2}-\ell_{1}\right)-\int_{0}^{\ell_{1}} \bar{C}_{A}(z, t) d z-\int_{\ell_{1}}^{\ell_{2}} \bar{C}_{A}(z, t) d z\right]
$$

\section{NUMERICAL RESOLUTION}

Drug distribution in the polymeric devices can be obtained by solving the diffusion Eq. (4), or Eqs. (10) and (11), depending on the number of layers of the platform. In this work, a simple numerical method, based on explicit finite differences, was used to obtain an approximation of drug concentration distribution at each desired instant of time.

The derivatives presented in Eq. (4) are approximated by using a central difference formula of second order to discretize the second order derivative on space (inside the polymeric layers, excluding the boundaries) and the Euler method of first order to discretize the first order derivative on time. Similar approximations were made to discretize Eqs. (10) and (11). The implementation of the numerical method was made using MATLAB software and all simulations are computed with this program.

Finally, the trapezoidal rule is used to approximate the integrals presented in Eqs. (9) and (19), in order to obtain the total mass of drug released, respectively, from one mono-layered patch and from one double-layered patch.

Parametric analysis is an excellent way to gain insight about the influence of parameters on drug delivery device performance. This information is a valuable tool to make decisions during product conception and development. Moreover, makes easier the answers to "what if" type questions.

The solution of the equations governing drug transport through a transdermal drug device provided at section 3.1 and 3.2 were numerically obtained with a MATLAB code, as referred before. This demands the assessment of a number of physical parameters. As the main goal of this work was to explore models' output sensitivity to some parameters characterizing drug dosage and transport through the patch, the parameters have been chosen as numeric values not in accordance with real situations. Whenever possible, the values attributed to the parameters belongs to the interval $[0,1]$ and are not followed by respective units.

In the simplest methodology used in parametric analysis, one parameter at a time is repeatedly varied while the others remain fixed. 
Table 1 shows the parameters values used to obtain qualitative predictions from the models proposed. Later, at sections 5.1 and 5.2, three alternative values for each parameter varied was considered: a low, medium and high value. In those sections, the drug profiles predicted after application of the patch loaded with drug in the skin are presented and discussed qualitatively, considering a mono and double-layered device.

Table 1 Parameters used to obtain the predictions in the transdermal drug devices. The parameters are numerical values without physical significance and for that reason units are not represented.

\begin{tabular}{|c|c|c|}
\hline \multirow{3}{*}{$\begin{array}{l}\text { drug diffusion coefficient in } \\
\text { the patch }\end{array}$} & \multicolumn{2}{|c|}{ mono-layered $\quad D=0.1$} \\
\hline & \multirow{2}{*}{ double-layered } & $\bar{D}=0.1$ \\
\hline & & $\overline{\bar{D}}=0.1$ \\
\hline \multirow{3}{*}{ patch thickness } & mono-layered & $\ell=1$ \\
\hline & \multirow{2}{*}{ double-layered } & $\ell_{1}=0.5$ \\
\hline & & $\left(\ell_{2}-\ell_{1}\right)=0.5$ \\
\hline \multirow{3}{*}{$\begin{array}{l}\text { initial drug concentration in } \\
\text { the patch }\end{array}$} & mono-layered & $C_{A i}=3$ \\
\hline & \multirow{2}{*}{ double-layered } & $\bar{C}_{A i}=3$ \\
\hline & & $\overline{\bar{C}}_{A i}=3$ \\
\hline $\begin{array}{l}\text { drug concentration in the patch } \\
\text { surface contacting skin }\end{array}$ & \multicolumn{2}{|l|}{$C_{\mathrm{As}}=\overline{\bar{C}}_{A s}=0$} \\
\hline $\begin{array}{l}\text { drug partition coefficient } \\
\text { between patch layers }\end{array}$ & \multicolumn{2}{|l|}{$k=1$} \\
\hline time & \multicolumn{2}{|l|}{$t_{f}=30$} \\
\hline space step & \multicolumn{2}{|l|}{$\Delta z=0.01$} \\
\hline time step & \multicolumn{2}{|l|}{$\Delta t=5 \times 10^{-5}$} \\
\hline
\end{tabular}

As well known by the literature, although the explicit method is of straightforward application and resolution it has a limitation on the size of the time step $\Delta t$. The stability condition $\Delta t<0.5 \Delta z^{2} / D$ is required.

\section{PREDICTIONS}

In all the situations considered to obtain predictions of drug delivery from patches, both mono and double-layered, the amount of drug incorporated in the device was assumed the same in order to facilitate the transdermal drug device performance comparison. In fact, patches that can provide extended medication release duration, at therapeutic doses, is one of the main goals in consideration when developing a new product.

\subsection{Transdermal drug device: mono-layered patch}

Figure 2 presents drug distribution inside a mono-layered patch after different periods of time of being applied to the skin. All the parameters and conditions used to obtain predictions are indicated in Table 1 for the mono-layered patch case. As expected, drug concentration inside the patch, for specific locations, decreases with time due to medication absorption through skin. However, higher drug concentrations, in each instant, is observed near the impermeable boundary (at $z=0$ ) and for small periods of time $(\leq 0.5)$ the initial concentration still exists in regions sufficiently far away from the surface in contact with skin. As a zero-sink boundary condition was used at $z=\ell$, drug concentration in that location is 0 during all delivering process. Once the patch is applied to the skin, a drug concentration gradient is generated in that location, resulting drug transfer by diffusion towards the patch surface where instantaneously disappears by skin absorption. Gradually, drug concentration gradients with decreasing magnitude are developed in regions progressively more distant from $z=\ell$. As the driving force for drug diffusion through transdermal device decreases in intensity, the amount of drug delivered to blood stream, through the skin, diminished in proportion, after the initial burst, as will be discussed in section 6 .

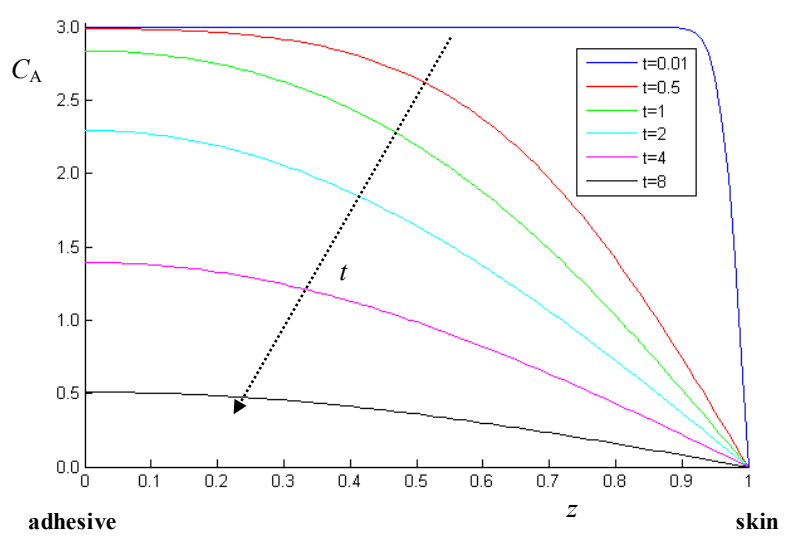

Fig. 2 Prediction of drug concentration inside the mono-layered patch $(\ell=1)$, for $t=0.01,0.5,1,2,4$ and 8 .

\subsection{Transdermal drug device: double-layered patch}

Drug distribution inside a double-layered patch was also predicted for different periods of time after application to the skin, using the numerical methodology described for the mathematical approach presented in section 3.2. All the parameters and conditions used are indicated in Table 1 , for the double-layered patch case, and the results obtained are shown in Fig. 3.

As the parameters and conditions introduced as input to obtain the predictions for the double-layered patch case are essentially the ones used for the mono-layered patch, as can be inspected by Table 1, the drug concentration distributions presented in Fig. 2 and Fig. 3 are coincident. In fact, the same total thickness for both patches were used, but now the patch is made with two different materials with the same thickness $(0.5)$ and equal amount of drug embedded ( $\bar{C}_{A i}=\overline{\bar{C}}_{A i}=3$ ). Moreover, it was assumed a particular situation where the two layers had the same ability to diffuse the drug, presenting equal values of diffusion coefficients ( $\bar{D}$ $=\overline{\bar{D}}=0.1)$.

This procedure was implemented as a simple strategic way to confirm if the new MATLAB code developed worked well. As the superposition of drug concentration distributions within mono and double-layered patches predicted with similar parameters and conditions was observed, in section 6 the same MATLAB code will be used to obtain more predictions. In that section, it will be modified individually the initial drug concentration within the two layers, as well as, the diffusion coefficients.

\section{PARAMETRIC EXPLORATION}

Sensitivity analysis of model parameters is an efficient way to gain valuable insights in drug mass transfer mechanisms throughout delivery devices and systematic exploration can be used to assess drug vehicles conception and optimization. Moreover, in vitro and in vivo studies will be considerably diminished after identification of dominant factors in the delivery process and establishment of conditions to obtain the therapeutic dosing interval. 
A model parameter sensitivity analysis was carried out for the mathematical approach presented to describe drug delivery from a transdermal device in order to identify the main parameters influencing the process. This analysis is particularly useful in two, or more, layered patches, where the parameter combination possibilities are enormous.

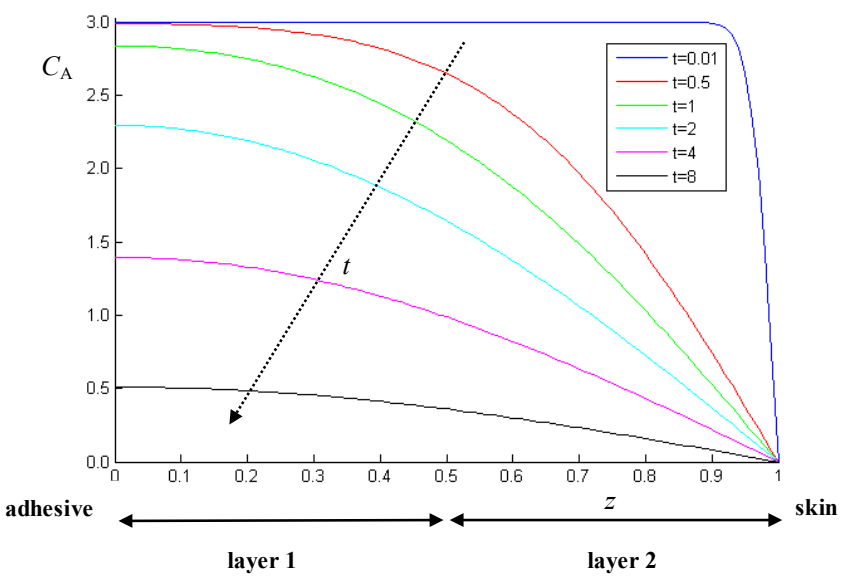

Fig. 3 Prediction of drug concentration inside the double-layered patch $\left(\ell_{1}=\left(\ell_{2}-\ell_{1}\right)=0.5\right)$, for $t=0.01,0.5,1,2,4$ and 8 .

A number of simulations with different values of drug diffusion coefficient were performed in order to quantify the effect of this parameter on the kinetics of drug delivery from a therapeutic monolayered patch. A two-fold variation of the diffusion coefficient, above and below its initial value (assumed equal to 0.1), was used in this evaluation. After a period $t=1$ of a mono-layered patch being applied to the skin, the distribution of the drug remaining inside was obtained with the three different values of the diffusion coefficient and are presented in Fig. 4 a). As expected, when a higher diffusion coefficient was used to obtain predictions, the drug concentration in all locations inside the patch is smaller enlightening that the delivery device has a greater ability to transport the drug from high concentration locations to the low concentration region, near the patch surface in contact with the skin. Hence, for that situation the transdermal device will be depleted in drug faster as it is shown in Fig. $4 \mathrm{~b}$ ). After a period $t=10$ the patch with $D=$ 0.2 have no drug inside and a period of $t>30$ is necessary for the patch with $D=0.05$ to deliver all the amount of drug loaded.

Transdermal patches are designed to slowly deliver active substances for long periods and maintain an appropriate therapeutic level. To ensure the effective use of these devices, some properties should be tested and one of the most important product descriptions is the strength, given as the mean mass of drug delivered per unit time, which is usually obtained measuring the dose delivered during 24 hours.

Using a matrix to support the active substance which provides a small drug diffusion coefficient will be more adequate if long periods to replace the patch are the objective, as can be seen from the dissolution profiles presented in Fig. 4 b), because lower drug delivery rates were observed. As expected, the drug dissolution profile is sensible to the diffusion coefficient value considered to represent the active substance transport parameter through the transdermal delivery device in the model proposed. For that reason, this will be a parameter modified later when performing the parametric sensitivity analysis in two-layered patches.

A global sensitivity analysis was also performed for therapeutic double-layered patches in which concerns the active substance concentration and its diffusion coefficient in both layers. Therefore, the effect of modifying each parameter in the predictions obtained with the model described in section 3.2, was explored individually. First, maintaining the initial dose of the active substance embedded in the patch equal to 3 , its concentration was modified in layer 1 and layer 2 attached to the skin. Secondly, the effect of considering different drug diffusion coefficients, in both layers, on the drug distribution in the device and on the dissolution profile, was carried out.

a)

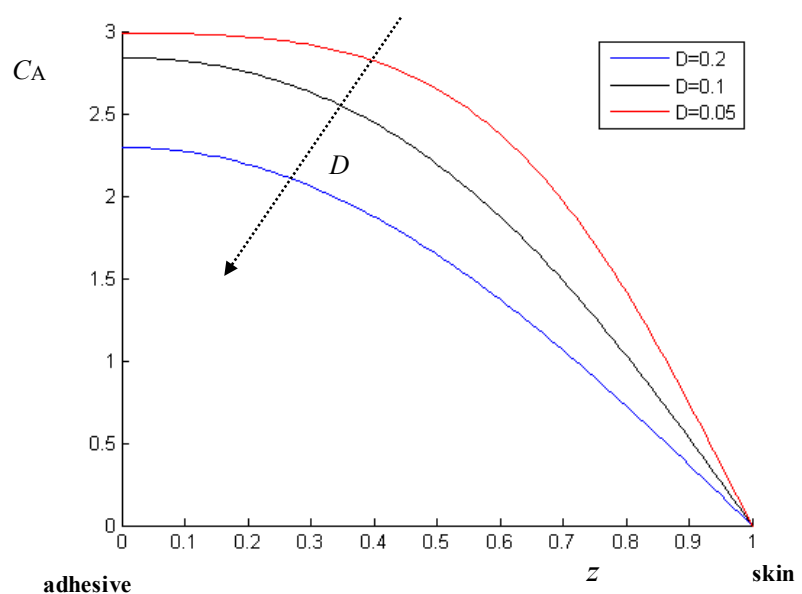

b)

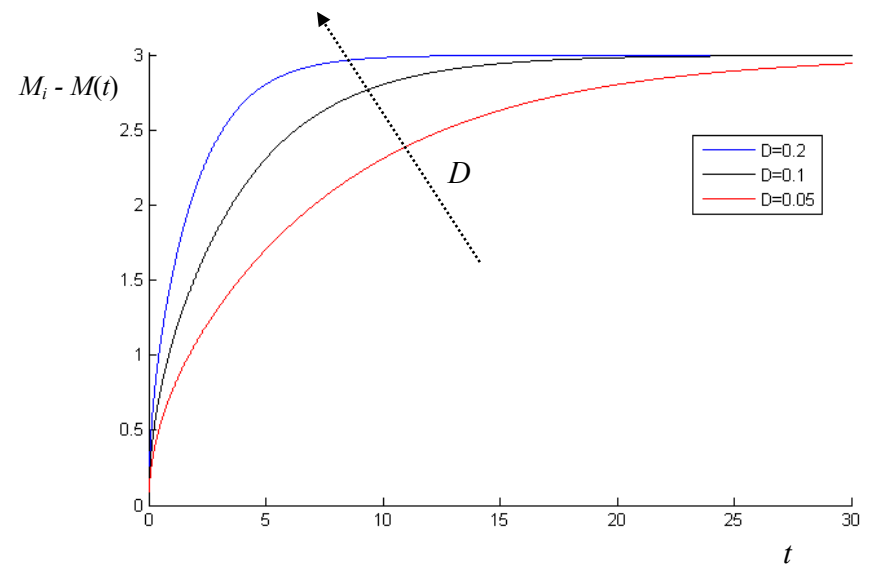

Fig. 4 Prediction of: (a) drug concentration inside the delivery device for $t=1$, and (b) drug release profile from a mono-layered patch $(\ell=1)$, for $D=0.05,0.1$ and 0.2 .

In Fig. 5 several drug concentration distributions inside the doublelayered patch, corresponding to different instants of time $(t=0.01,0.5$, $1,2,4$ and 8) are represented for four initial concentration of the active substance within the transdermal device. The situation represented in Fig. $5 \mathrm{a}$ ), with initial drug concentration uniform in both layers, will serves as reference for the other cases where layer 1 and layer 2 started the delivering process with different drug concentrations. When the initial drug concentration is lower in layer 2, in contact with the skin, the concentration gradient developed when the patch is applied have a lower magnitude and the initial burst of drug delivered is not so intense. It should be noted that in order to maintain the amount of active substance occluded in the therapeutic device equal to 3 , if $\bar{C}_{A i}=4$ is the initial drug concentration in layer 2 must be $\overline{\bar{C}}_{A i}=2$, as the total patch volume is divided in equal parts of layer 1 and 2 . Conversely, if the initial drug concentration in layer 2 is increased to $\overline{\bar{C}}_{A i}=4$, in layer 1 must decrease to $\bar{C}_{A i}=2$, to have an amount of medication of 3 embedded in the patch. This situation will enhance the initial "explosion" of drug from the 
a)

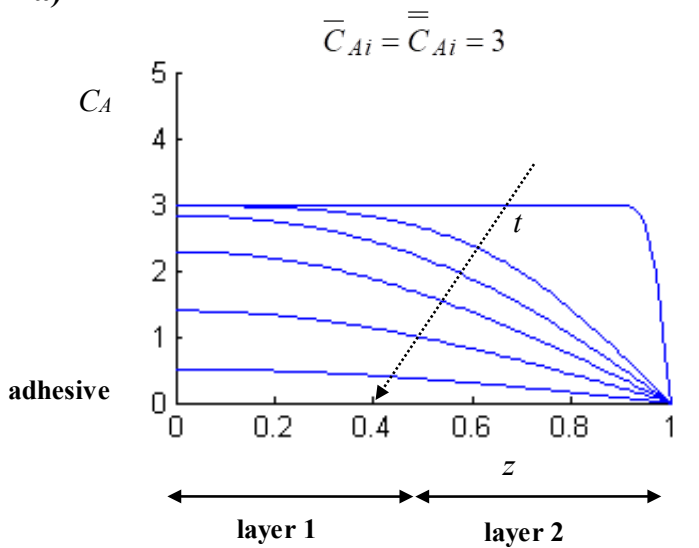

c)

$$
\bar{C}_{A i}=5 \quad \bar{C}_{A i}=1
$$

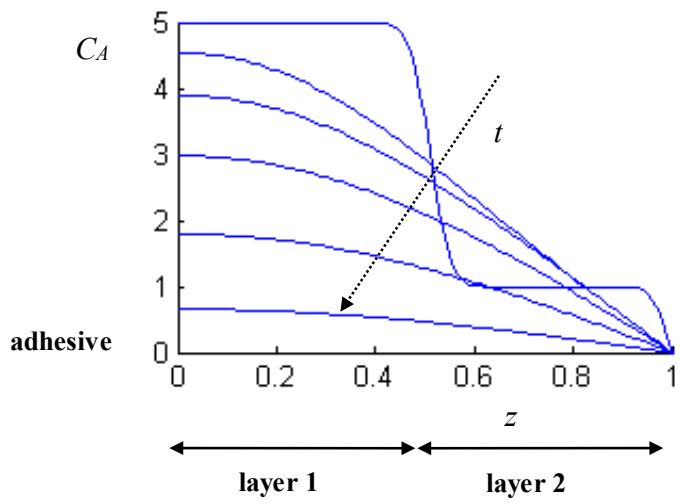

b)

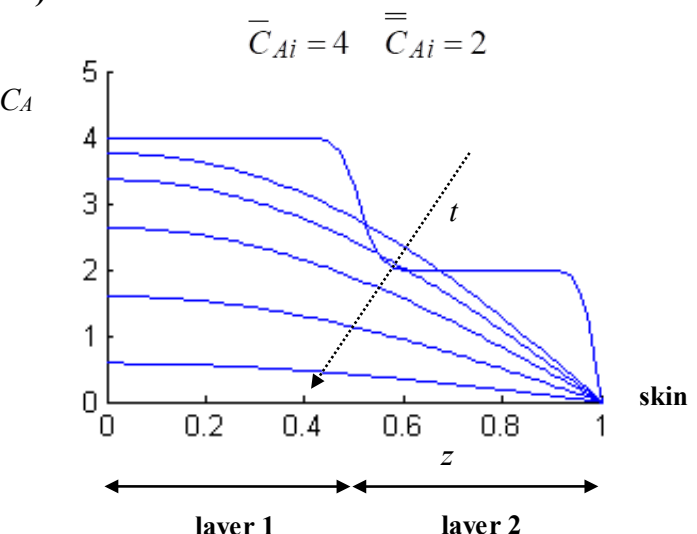

d)

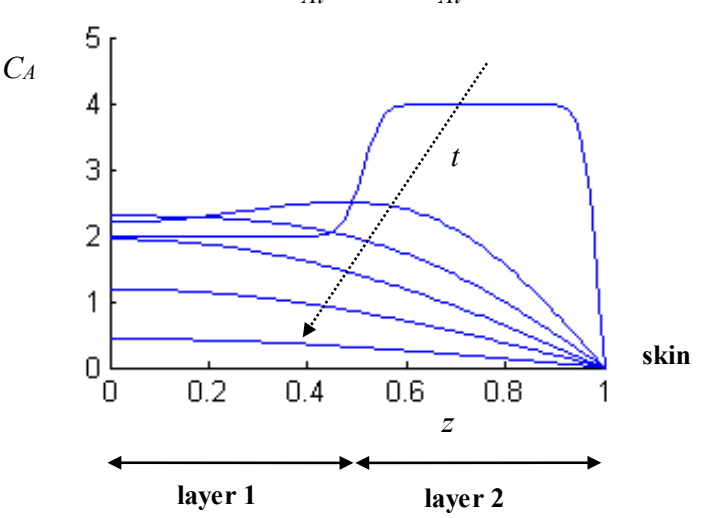

Fig. 5 Prediction of drug concentration distribution inside the double-layered patch $\left(\ell_{1}=\left(\ell_{2}-\ell_{1}\right)=0.5\right)$, for $t=0.01,0.5,1,2,4$ and 8 and for different initial doses of drug added into the layers. a) $\bar{C}_{A i}=\overline{\bar{C}}_{A i}=3 ;$ b) $\bar{C}_{A i}=4$ and $\overline{\bar{C}}_{A i}=2$; c) $\bar{C}_{A i}=5$ and $\bar{C}_{A i}=1$ and d) $\bar{C}_{A i}=2$ and $\overline{\bar{C}}_{A i}=4$.

transdermal device, as can be seen in Fig. 6, because the concentration gradient in the patch at the skin surface is higher (see Fig. 5 d)).

In fact, the dissolution profiles shown in Fig. 6 reveal that a multilayer transdermal device could be designed with the intent to tune the drug release. The initial drug concentration inside the layer attached to the skin (layer 2) dictates the immediate release and the other layer serves to control the release of drug during large periods. An adequate design of multilayer devices can be used to regulate the kinetics of drug release and optimize the therapeutic response. To extend the transdermal patch duration safe and effectively, it should not irritate the skin and the medication should be delivered through the skin at an adequate rate. When low initial contents of drug in the layer attached to the skin was used, it favors a moderate release rate of the active substance since the moment the patch is applied, increasing the period that it could remain in place with therapeutic benefits. In fact, an efficient tuning of drug release decreases the amplitude of drug concentration, avoiding peaks of concentration and long periods of sub-therapeutic level. As a result, the period that patch remains in place can be increased as the active substance concentration with an effective level at the intended location for treatment will exists during long periods.

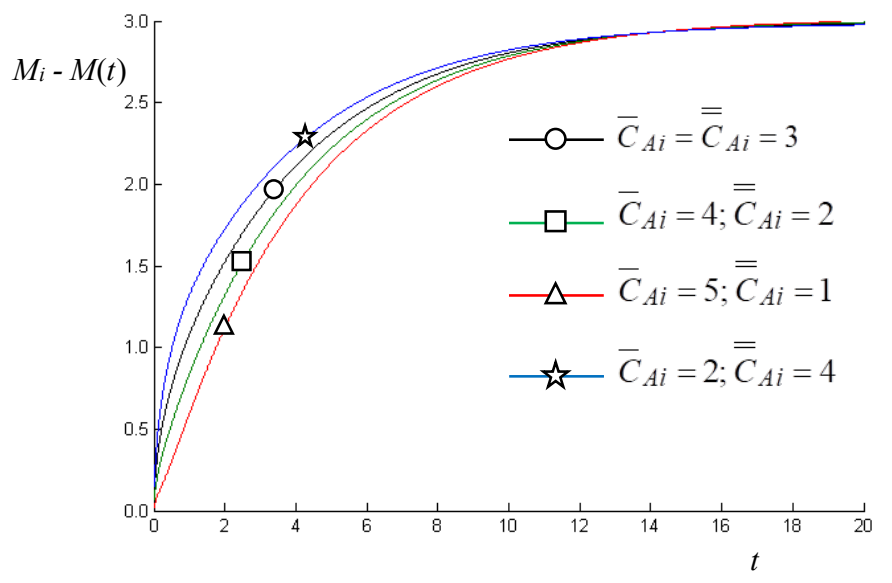

Fig. 6 Prediction of drug release profile from a double-layered patch $\left(\ell_{1}=\left(\ell_{2}-\ell_{1}\right)=0.5\right)$ for different initial doses of drug added into the layers. 
Continuing with the purpose of extending patch life with therapeutic effects, three different simulations were performed varying, individually, below the reference value of $D=0.1$, the diffusion coefficient for layer 1 and layer 2 of the two-layered patch in consideration. Decreasing the reference value is in accordance with the main conclusions coming from the diffusion coefficient effect on drug distribution in the mono-layered patch discussed before in this section.

Next figure (Fig. 7) shows the drug concentration distribution obtained for four different conjugations of diffusion coefficient values in both layers and for several instants of time $(t=0.01,0.5,1,2,4$ and 8$)$. All the predictions in the figure were obtained for the same initial drug concentration condition in both layers, $\bar{C}_{A i}=\overline{\bar{C}}_{A i}=3$. The first scenario, where the diffusion coefficient is the same in both layers and equal to 0.1 , serves as reference for the others situations considered. As can be seen from Fig. $7 \mathrm{~b}$ ) and c), when the diffusion coefficient for layer 1 and layer 2 decreases, the drug concentration level remaining within the patch is higher, which is more noticeable for greater instants of time. However, it should be emphasized that the effect is more pronounced when the ability to diffuse the drug is diminished in the layer in contact with the skin (layer 2). In fact, the highest drug concentration gradients in the patch occur near the skin and if in that location the diffusion coefficient is low, is more effective the slowing down of drug release. Figure $7 \mathrm{~d}$ ), presenting drug concentration distribution in the patch obtained with $\overline{\bar{D}}$ even smaller, shows that diffusion of drug is so much slower that the decrease in concentration in layer 1 from its initial value is only noticeable for instants of time higher than $t=2$. As a consequence the active substance concentration within layer 2 is much higher, being drug release rate more moderate, essentially at the beginning of the releasing process.

Dissolution profiles presented in Fig. 8 are the consequence of drug concentration distribution developed within both patch layers shown in Fig. 7 for different scenarios of the ability of media to diffuse the active substance. Using the medium with lower drug diffusion coefficient to make the patch layer in contact with the skin is a good option to extend the drug release, as can be observed from Fig. 8. In order to slow down the initial burst of drug, its diffusion coefficient in layer 2 near the skin must be decreased, although the therapeutic level must be guaranteed during considerable periods to the patch be effective in treatment.

b)

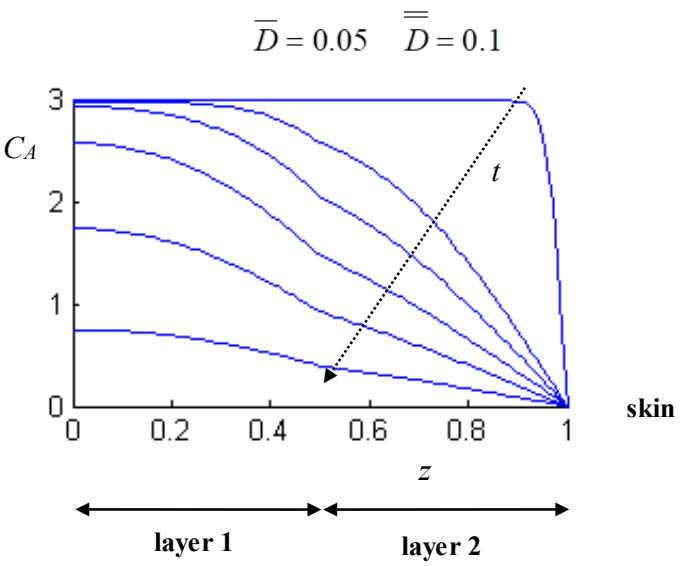

d)

c)

$$
\bar{D}=\overline{\bar{D}}=0.1
$$

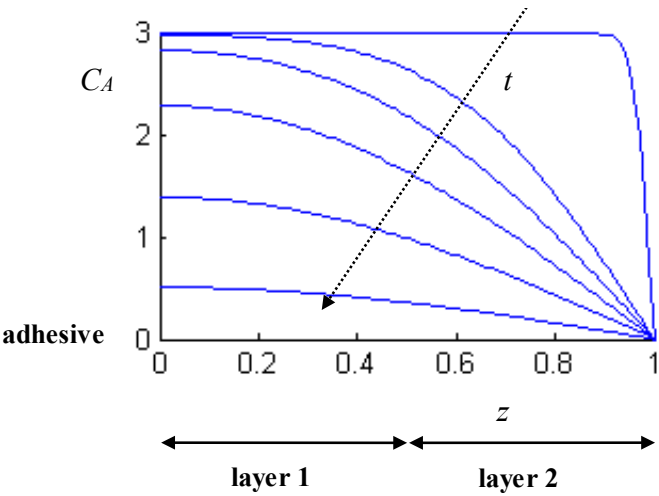

$$
\bar{D}=0.1 \quad \overline{\bar{D}}=0.05
$$

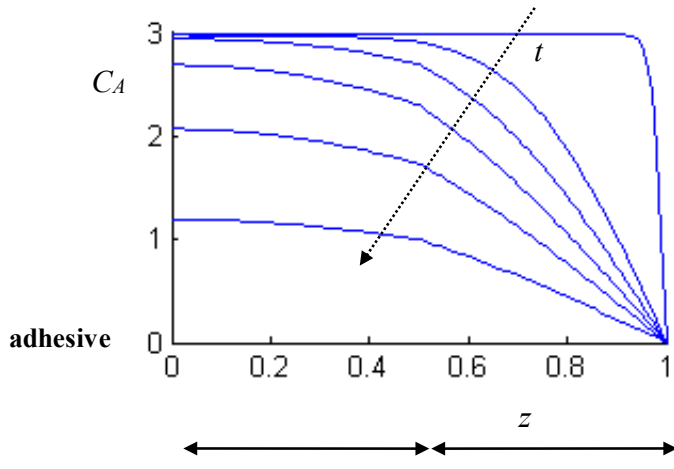

layer 1

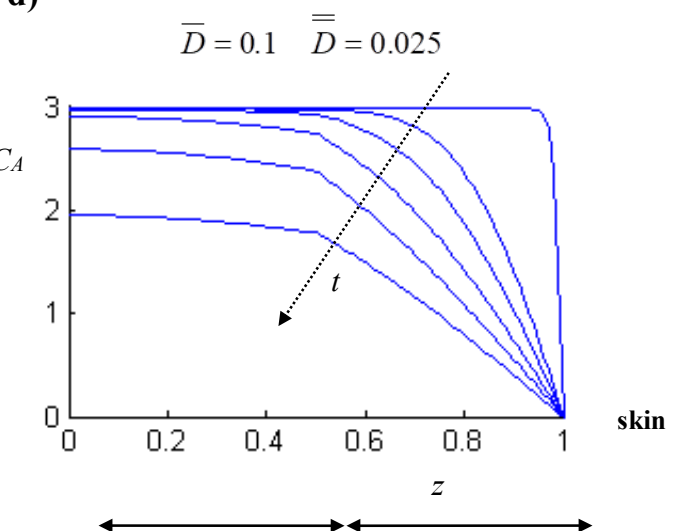

layer 1

layer 2

Fig. 7 Prediction of drug concentration distribution inside the double-layered patch $\left(\ell_{1}=\left(\ell_{2}-\ell_{1}\right)=0.5\right)$, for $t=0.01,0.5,1,2,4$ and 8 and for different diffusion coefficient in both layers. a) $\bar{D}=\overline{\bar{D}}=0.1$; b) $\bar{D}=0.05$ and $\overline{\bar{D}}=0.1 ; \bar{D}=0.1$ and $\overline{\bar{D}}=0.05$ and d) $\bar{D}=0.1$ and $\overline{\bar{D}}=0.025$. 


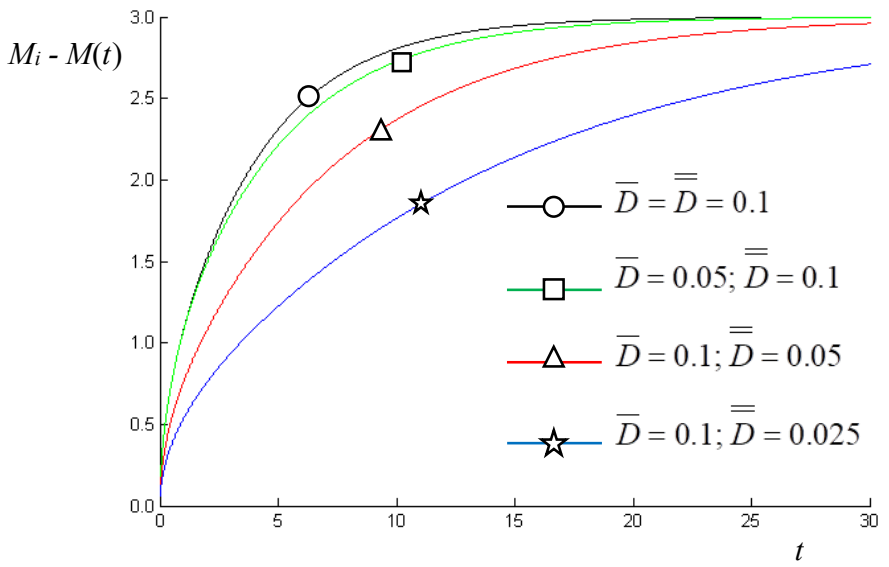

Fig. 8 Prediction of drug release profile from a double-layered patch $\left(\ell_{1}=\left(\ell_{2}-\ell_{1}\right)=0.5\right)$ with different diffusion coefficient values in both layers.

After exploring individually the effect of initial drug concentration and diffusion coefficient in both patch layers on model output giving drug distribution within the patch, two main conclusions can be highlighted:

- low initial active substance concentration within the patch layer in contact with the skin;

- low ability of the support material used in the patch layer in contact with the skin to diffuse the active substance;

both contribute to slow down the initial drug burst during the delivering process.

Using an optimal conjugation could improve even more the patch lifetime with therapeutic effects. Following this idea, a simulation with the lowest values of $\overline{\bar{C}}_{A i}$ and $\overline{\bar{D}}$ used in the individual parametric study, was performed and the resulting kinetics for drug delivery process is shown in the next figure (Fig. 9). To facilitate the comparison of varying both parameters at the same time, the dissolution profiles for: i) $\bar{D}=\overline{\bar{D}}$ $=0.1$ and $\bar{C}_{A i}=\overline{\bar{C}}_{A i}=3$ (the reference situation); ii) $\bar{D}=\overline{\bar{D}}=0.1$ and $\bar{C}_{A i}=5, \bar{C}_{A i}=1$ (the best scenario used in the study of initial drug concentration effect) and iii) $\bar{D}=0.1, \overline{\bar{D}}=0.025$ and $\bar{C}_{A i}=\overline{\bar{C}}_{A i}=3$ (the best situation used in the diffusion coefficient parametric study), are also represented in Fig. 9.

Analysing Fig. 9, it is clear that the conjugation of the two parameter values identified previously as the best to obtain moderate drug release rates at the beginning of the drug absorption process through the skin, favours even more the goal of enlarge patch life with therapeutic benefits.

The systematic parametric study developed, which culminated with predictions in Fig. 9 helped to understand and gain sensitivity to the importance of modelling in the design and conception of new products. Even though, in similar situations as the one described where numerical values without physical meaning were used for the parameters varied in the model input.

\section{CONCLUSIONS}

The goal of this study was develop simple models to describe the unsteady state diffusion of an active principle through a polymeric matrix constituting a transdermal device, in order to afterwards perform easily a parametric sensitivity analysis.

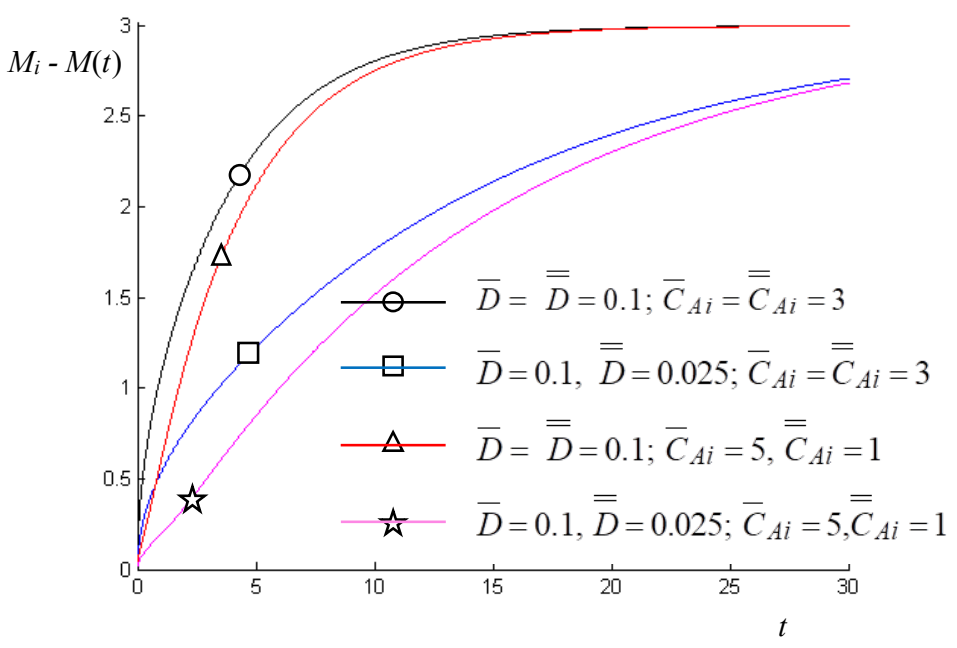

Fig. 9 Prediction of drug release profile from a double-layered patch $\left(\ell_{1}=\left(\ell_{2}-\ell_{1}\right)=0.5\right)$ for four different conjugations of diffusion coefficient values and initial doses of drug in both layers.

Transdermal patches made of a single adhesive material layer with the drug embedded and made of two different layers were considered for modelling drug release to the skin. Simultaneously, a parametric sensitivity analysis was carried out in order to evaluate crucial parameters in the transdermal devices optimization process. Low initial drug concentration and low ability to diffuse the active substance in the support material used to make the patch layer in contact with the skin favor the slowing down of the initial drug burst during the delivering process. Using the optimal conjugation of these key parameters the patch lifetime with therapeutic effects could be improved. The parametric exploration brought insight into the drug transfer process inside patches and showed it can be a useful tool to obtain optimized formulations.

\section{NOMENCLATURE}

$A$

polymeric patch area

$C_{A} \quad$ drug concentration in the solid matrix

$\bar{C}_{A} \quad$ drug concentration in layer 1 in a double-layered patch

$\overline{\bar{C}}_{A} \quad$ drug concentration in layer 2 in a double-layered patch

$C_{A i} \quad$ initial drug concentration in the delivery device

$\bar{C}_{A i} \quad$ initial drug concentration throughout layer 1 in a doublelayered patch

$\overline{\bar{C}}_{A i} \quad$ initial drug concentration throughout layer 2 in a doublelayered patch

$C_{A s} \quad$ drug concentration in the patch surface contacting the skin

$\overline{\bar{C}}_{A s} \quad$ drug concentration in the surface of layer 2 in a doublelayered patch, contacting the skin drug diffusion coefficient drug diffusion coefficient in layer 1 in a double-layered patch drug diffusion coefficient in layer 2 in a double-layered patch drug partition coefficient

patch thickness

$\ell_{1} \quad$ thickness of layer 1 in a double-layered patch

$\ell_{2} \quad$ total thickness of a double-layered patch

$M \quad$ amount of medication inside the patch 
$M_{i} \quad$ initial amount of medication in the patch

$t \quad$ time

$x \quad$ coordinate

$y \quad$ coordinate

z coordinate

Greek Symbols

$\Delta z \quad$ space step

$\Delta t \quad$ time step

\section{REFERENCES}

Anissimov, Y.G., Jepps, O.G., Dancik, Y. and Roberts, M.S., 2013, "Mathematical and Pharmacokinetic Modelling of Epidermal and Dermal Transport Processes," Advanced Drug Delivery Reviews, 65(2), 169-190.

http://dx.doi.org/10.1016/j.addr.2012.04.009

Frasch, H.F. and Barbero, A.M., 2013, "Application of Numerical Methods for Diffusion-Based Modeling of Skin Permeation," Advanced Drug Delivery Reviews, 65(2), 208-220. http://dx.doi.org/10.1016/j.addr.2012.01.001

Jepps, O.G., Dancik, Y., Anissimov, Y.G. and Roberts, M.S., 2013, "Modeling the Human Skin Barrier - Towards a Better Understanding of Dermal Absorption," Advanced Drug Delivery Reviews, 65(2), 152-168. http://dx.doi.org/10.1016/j.addr.2012.04.003
Kalia, Y.N. and Guy, R.H., 2001, "Modeling Transdermal Drug Release," Advanced Drug Delivery Reviews, 48(2-3), 159-172.

http://dx.doi.org/10.1016/S0169-409X(01)00113-2

Khanday, M.A. and Rafiq, A., 2015, "Variational Finite Element Method to Study the Absorption Rate of Drug at Various Compartments through Transdermal Drug Delivery System," Alexandria Journal of Medicine, 51(3), 219-223.

http://dx.doi.org/10.1016/j.ajme.2014.09.001

Pontrelli, G. and de Monte, F., 2014, “A Two-Phase Two-Layer Model for Transdermal Drug Delivery and Percutaneous Absorption," Mathematical Biosciences, 257(November), 96-103. http://dx.doi.org/10.1016/j.mbs.2014.05.001

Rim, J.E., Pinsky, P.M. and Van Osdol, W.W., 2005, "Finite Element Modeling of Coupled Diffusion with Partitioning in Transdermal Drug Delivery," Annals of Biomedical Engineering, 33(10), 1422-1438. http://dx.doi.org/10.1007/s10439-005-5788-6

Snorradõttir, B.S., Jõnsdõttir, F., Sigurdsson, S.TH. and Másson, M., 2014, "Numerical Modelling of Transdermal Delivery from Matrix Systems: Parametric Study and Experimental Validation with Silicone Matrices," Journal of Pharmaceutical Sciences, 103(8), 2366-2375. http://dx.doi.org/10.1002/jps.24052

Williams, A.C., 2003, Transdermal and Topical Drug Delivery: From Theory to Clinical Practice, $1^{\text {st }}$ ed., Pharmaceutical Press. 